\title{
A transferable ab-initio based force field for aqueous ions
}

\author{
Sami Tazi, John J. Molina, Benjamin Rotenberg, Pierre Turq, and Mathieu Salanne \\ UPMC Univ Paris 06, CNRS, ESPCI, \\ UMR 7195 PECSA, F-75005 Paris, France \\ Rodolphe Vuilleumier \\ UPMC Univ Paris 06, Ecole Normale Supérieure, \\ Département de Chimie, F-75231 Paris, France
}

\begin{abstract}
We present a new polarizable force field for aqueous ions $\left(\mathrm{Li}^{+}, \mathrm{Na}^{+}, \mathrm{K}^{+}, \mathrm{Rb}^{+}, \mathrm{Cs}^{+}, \mathrm{Mg}^{2+}\right.$, $\mathrm{Ca}^{2+}, \mathrm{Sr}^{2+}$ and $\mathrm{Cl}^{-}$) derived from condensed phase ab-initio calculations. We use Maximally Localized Wannier Functions together with a generalized force and dipole-matching procedure to determine the whole set of parameters. Experimental data is then used only for validation purposes and a good agreement is obtained for structural, dynamic and thermodynamic properties. The same procedure applied to crystalline phases allows to parametrize the interaction between cations and the chloride anion. Finally, we illustrate the good transferability of the force field to other thermodynamic conditions by investigating concentrated solutions.
\end{abstract}




\section{INTRODUCTION}

The development of classical force fields for ions in aqueous solution is essential to the description of specific effects, which are legion in biochemistry $\underline{\underline{1}}^{-\underline{3}}$, atmospheric chemistry $\underline{\underline{4}}^{\underline{4}}$ or environmental science ${ }^{5}$. The reliability of molecular simulations strongly depends on the quality of the force field used to represent the interactions, which must capture not only the effects of ionic size, but also the polarization of water by the ionic charge. The latter multi-body effect becomes essential when dealing with multivalent ions ${ }^{\underline{6}}$, in concentrated solutions $s^{7}$ and in interfacial environments $\frac{4,8,9}{2}$.

In recent years, a successful strategy to derive polarizable force fields for solid and molten oxides from ab-initio simulations has been developed by Madden and co-workers $\underline{10}-12$. A full set of parameters was obtained for the $\mathrm{Ca}-\mathrm{Mg}-\mathrm{Al}-\mathrm{Si}-\mathrm{O}$ (CMAS) system ${ }^{13}$, which is the main component of the Earth's crust and mantle. Cation-rich aluminosilicates, including clays and zeolites, are also the principal minerals on the Earth's surface, where they are in contact with ionic solutions. Examples of situations where the interface between such minerals and solution play an important role include the crystallization and dissolution of ionic crystals, such as calcium carbonate (in the context of carbon dioxide sequestration ${ }^{14}$ ) or sodium sulfate (deterioration of monuments $\frac{15}{}$ ), or the sorption of radioactive contaminants (e.g. cesium or strontium) onto clays $\frac{16}{27}$. It is therefore of primary importance to extend the CMAS force field in order to describe these minerals and their interaction with water and ions. As a first step in this direction, we develop here a polarizable force field for ions in water which is compatible with that developed for the CMAS system.

As mentioned previously, and despite the success of non-polarizable water force fields

in the bulk $k^{18-21}$, transferability to interfaces, especially charged ones, requires resorting to a polarizable model. Many such models exist, which differ mainly in their treatment of the polarizability. Drude or shell models assign a charge on a spring to each polarizable atom $22-24$. Other approaches allow for charge fluctuations $\underline{25}, 26$ or assign point dipoles to each polarizable species ${ }^{27}-31$. Only the latter model is compatible with the above-mentioned one for oxides. Among the point polarizability models, we chose the one of Dang and Chang which was specifically developed to describe the gas-liquid interface 29 . In addition, Masia et al. have shown that it accurately reproduces the strong water polarization by divalent cations $^{32,33}$. 
Following the strategy of Madden and co-workers, which has proven able to simultaneously reproduce structural, dynamic and thermodynamic properties not only for the CMAS system, but also for many other ionic materials $11,12,34,35$, we derive here the parameters of a force field for the aqueous ions: $\left(\mathrm{Li}^{+}, \mathrm{Na}^{+}, \mathrm{K}^{+}, \mathrm{Rb}^{+}, \mathrm{Cs}^{+}, \mathrm{Mg}^{2+}, \mathrm{Ca}^{2+}, \mathrm{Sr}^{2+}\right.$ and $\mathrm{Cl}^{-}$. The route from condensed phase Density Functional Theory (DFT) calculations, using Max-

imally Localized Wannier Functions (MLWFs) $\underline{36}, \frac{37}{6}$ together with a generalized force and dipole-matching procedure ${ }^{38} \underline{39}$, renders experimental input unnecessary, contrary to many force field parametrizations.

The paper is organized as follows: We first give a detailed description of the force field and its parametrization which involves DFT calculations on single ions in bulk water and on ionic crystals. The second part is then devoted to the validation of the model, against structural, dynamic and thermodynamic properties of these systems. Finally, the transferability of the model is illustrated by the study of concentrated salt solutions.

\section{THE FORCE FIELD AND ITS PARAMETRIZATION}

\section{A. Model}

The total energy of the system is decomposed into four terms:

$$
V_{\text {tot }}=V_{\text {charge }}+V_{\text {disp }}+V_{\text {rep }}+V_{\text {pol }}
$$

For the calculation of the direct Coulomb interaction between two atoms $I$ and $J$,

$$
V_{\text {charge }}=\sum_{I, J>I} \frac{q^{I} q^{J}}{r_{I J}}
$$

formal charges (here $-1,+1$ or +2 ) are used. The dispersion potential includes the dipoledipole and dipole-quadrupole terms

$$
V_{\mathrm{disp}}=-\sum_{I, J>I}\left[f_{6}^{I J}\left(r_{I J}\right) \frac{C_{6}^{I J}}{r_{I J}^{6}}+f_{8}^{I J}\left(r_{I J}\right) \frac{C_{8}^{I J}}{r_{I J}^{8}}\right]
$$

and the short-range corrections are described using the Tang-Toennies functions $f_{n}^{I J}$, which are of the form $\underline{40}$ :

$$
f_{n}^{I J}=1-e^{-b_{D}^{I J} r_{I J}} \sum_{k=0}^{n} \frac{\left(b_{D}^{I J} r_{I J}\right)^{k}}{k !}
$$


While the repulsion potential is modelled using a decaying exponential:

$$
V_{\text {rep }}=\sum_{I, J>I} A^{I J} e^{-B^{I J} r_{I J}}
$$

Finally, many-body electrostatic effects are described by the induced dipoles $\mu^{I}$, which are treated as additional degrees of freedom and obtained at each MD step by minimizing the polarization energy:

$$
V_{\mathrm{pol}}=\sum_{I} \frac{1}{2 \alpha^{I}}\left|\mu^{I}\right|+\sum_{I, J}\left[\left(q^{I} \mu_{\alpha}^{J} g^{I J}\left(r_{I J}\right)-q^{J} \mu_{\alpha}^{I} g^{J I}\left(r_{I J}\right)\right) T_{I J}^{\alpha}-\mu_{\alpha}^{I} \mu_{\beta}^{J} T_{I J}^{\alpha \beta}\right]
$$

with $\alpha^{I}$ the ion polarizability and where the Einstein summation convention is assumed. A short-range correction to the multipolar expansion of the Tang-Toennies type is used:

$$
g^{I J}\left(r_{I J}\right)=1-c^{I J} e^{-b^{I J} r_{I J}} \sum_{k=0}^{4} \frac{\left(b^{I J} r_{I J}\right)^{k}}{k !}
$$

This so-called Polarizable Ion Model (PIM) has proven extremely successful for the description of oxides, chloride and fluoride-based materials, both in the solid and liquid states $11,12,34$. Water is described by a model compatible with this form, developed by Dang and Chang 29 . The only differences with the PIM are the description of the repulsive and dispersion terms $V_{\text {rep }}+V_{\text {disp }}$ for the water-water interactions, represented by a Lennard-Jones potential, and the absence of short-range damping of the charge-dipole interaction. The Dang-Chang (DC) water is a rigid 4-site model, with an additional virtual site $\mathrm{M}$ along the symmetry axis of the molecule, which bears a negative partial charge, as well as the induced dipole, while the Lennard-Jones interaction acts on the oxygen atom only. The parameters of the DC model are summarized in Table —.

\begin{tabular}{|c|c|c|c|c|c|c|}
\hline $\mathrm{d}_{\mathrm{OH}} \AA$ & $\mathrm{d}_{\mathrm{OM}} \AA$ & angle $\left(^{\circ}\right)$ & $\epsilon_{\mathrm{O}}(\mathrm{kcal} / \mathrm{mol})$ & $\sigma_{\mathrm{O}} \AA$ & $\mathrm{q}_{\mathrm{H}}$ & $\alpha_{\mathrm{M}} \AA$ \\
\hline \hline 0.9752 & 0.215 & 104.52 & 0.1825 & 3.2340 & 0.5190 & 1.444 \\
\hline
\end{tabular}

TABLE I: Parameters of the Dang-Chang water model.

The purpose of the present work is to derive all the parameters of the PIM for water-ion and ion-ion interactions, thereby providing a force field for the simulation of ions which is transferable from infinite dilution to concentrated solutions, up to the ionic crystals, for alkaline $\left(\mathrm{Li}^{+}, \mathrm{Na}^{+}, \mathrm{K}^{+}, \mathrm{Rb}^{+}, \mathrm{Cs}^{+}\right)$and alkaline earth $\left(\mathrm{Mg}^{2+}, \mathrm{Ca}^{2+}, \mathrm{Sr}^{2+}\right)$ cations and the 
chloride $\left(\mathrm{Cl}^{-}\right)$anion. Overall, this requires specifying 241 parameters. The procedure to determine all of them from ab-initio calculations aims at minimizing the risk of compensation of errors among the different terms by 1) directly computing as many parameters as possible, 2) adjusting the remaining ones on different quantities (dipoles and forces) and 3) resorting to simplifying assumptions when necessary. We now describe these three aspects.

\section{B. Calculating parameters}

First-principle calculations based on Density Functional Theory (DFT) describe the electronic density using the Kohn-Sham orbitals, whose delocalized nature renders the assignment of atomic or molecular properties difficult. The concept of the maximally localized Wannier function (MLWF) provides a convenient framework to analyze atomic and molecular properties in the condensed phase ${ }^{41}$. The Wannier functions are defined through a unitary transformation of the Kohn-Sham eigenvectors. MLWFs are contructed by choosing the phase so that it minimizes the spread of the Wannier function $\stackrel{41}{ }$. It was shown recently that MLWFs could be used to systematically derive both the polarizabilities $\alpha^{I}$ and dispersion parameters $C_{6}^{I J}$ and $C_{8}^{I J}$ of a PIM ${ }^{37} \underline{39}$. Figure 1 illustrates the electronic density around a $\mathrm{Ca}^{2+}$ cation and two water molecules in bulk water, reconstructed from their respective Wannier orbitals.

\section{Polarizability}

In a closed shell system, each MLWF contributes two electrons, so that the atomic or molecular dipole can be computed (in atomic units) as

$$
\boldsymbol{\mu}^{I}=\sum_{i \in I}\left(Z_{i} \boldsymbol{R}_{i}-2 \sum_{n \in i} \boldsymbol{r}_{n}^{w}\right)
$$

where the sums run over atoms $i$ belonging to fragment $I$ and over MLWFs $n \in i$ whose center is localized in the vicinity of the nuclear position $\boldsymbol{R}_{i}, Z_{i}$ is the charge of nucleus $i$ and $r_{n}^{w}$ is the position of the center of the $n$-th MLWF. The polarizability may differ from that of the same ion in the gas phase because of environmental effects. It can be calculated by applying a small electric field $\mathcal{E}^{(\alpha)}$ along each Cartesian direction $\alpha=x, y, z$ to the system,

which induces dipole moments $\left\{\delta \boldsymbol{\mu}^{I,(\alpha)}\right\}_{I \in[1, N]}$. A convenient way to distinguish the effect of 


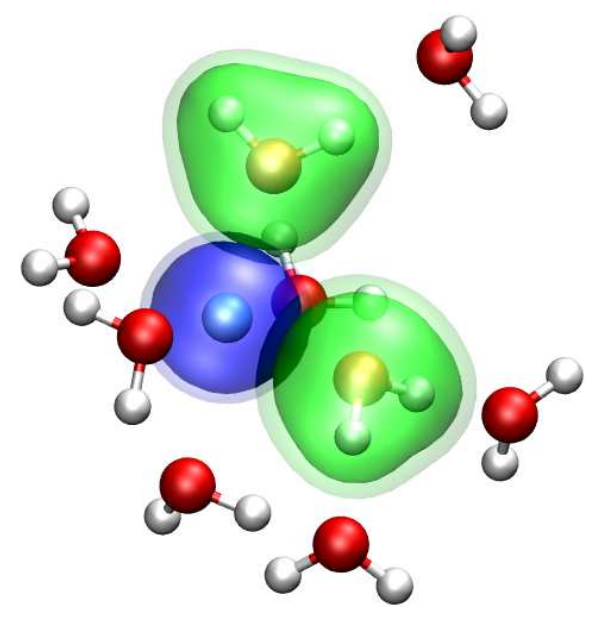

FIG. 1: Localized electronic density around a $\mathrm{Ca}^{2+}$ cation and two water molecules in bulk water, reconstructed from their respective Wannier orbitals. The isodensity surfaces include $90 \%$ and $95 \%$ of the corresponding densities, respectively.

the applied field from that of the static fields caused by the permanent charge distributions of the molecules, is to think of the former as an optical field. $\delta \mu^{I}$ can then be seen as the net induced dipole oscillating at the optical frequency. The total field $\boldsymbol{f}^{I,(\alpha)}$ on each atom is

$$
\boldsymbol{f}^{I,(\alpha)}=\mathcal{E}^{(\alpha)}+\sum_{J \neq I} \boldsymbol{T}^{I J} \cdot \delta \boldsymbol{\mu}^{J}\left(\boldsymbol{R}^{N}\right)
$$

where $\boldsymbol{T}^{I J}$ is the dipole-dipole interaction tensor. The polarizability tensor of molecule $I$ can then be obtained by inverting Eq. (9) :

$$
\boldsymbol{\alpha}^{I}\left(R^{N}\right)=\left(\boldsymbol{F}^{I}\right)^{-1} \cdot \Pi^{I}
$$

with the second-rank three dimensional tensors defined as : $F_{\alpha \beta}^{I}=f_{\alpha}^{I,(\beta)}$ and $\Pi_{\alpha \beta}^{I}=\delta \mu_{\alpha}^{I,(\beta)}$ More details about this approach can be found in Ref. $\underline{\underline{42}}$. 


\section{Dispersion: $C_{6}$ and $C_{8}$}

DFT calculations do not usually account for dispersion interactions, because the former describe the electronic ground state while the latter arise from correlated density fluctuations associated with excited states. The treatment of dispersion via non-local functionals has only recently been introduced, albeit at a high computational cost. Thus these interactions are generally added (if at all) as an a posteriori correction. Among the several methods that have been proposed for computing this correction, the method of Grimme ${ }^{43}$ and that of Silvestrelli $\frac{44}{-}$ seem to be the most popular. In this work we use the latter, which considers the dispersion interaction between all pairs of MLWF as follows. The long-range interaction between separated fragments of matter is calculated, following Andersson et al. $\underline{45}$, as

$$
E_{x c}^{l r}=\frac{6 e}{4(4 \pi)^{3 / 2} m^{1 / 2}} \iint_{V_{1} V_{2}} \mathrm{~d} \boldsymbol{r}_{1} \mathrm{~d} \boldsymbol{r}_{2} \frac{\sqrt{\rho_{1}\left(\boldsymbol{r}_{1}\right) \rho_{2}\left(\boldsymbol{r}_{2}\right)}}{\sqrt{\rho_{1}\left(\boldsymbol{r}_{1}\right)}+\sqrt{\rho_{2}\left(\boldsymbol{r}_{2}\right)}} \times \frac{1}{\left\|\boldsymbol{r}_{1}-\boldsymbol{r}_{2}\right\|^{6}}
$$

where $\rho\left(r_{i}\right)$ is the charge density of fragment $i, m$ the electron mass and $V_{i}$ the volume occupied by fragment $i$. For large separations $R$, this scales as $E^{l r}=-C_{6} / R^{6}$, where the $C_{6}$ coefficient for the interaction between two MLFWs $k$ and $l$ can be computed as :

$$
C_{6}^{k l}=\frac{3}{32 \pi^{3 / 2}} \iint_{\substack{r_{1} \leq r_{c} \\ r_{2} \leq r_{c}^{\prime}}} \mathrm{d} \boldsymbol{r}_{1} \mathrm{~d} \boldsymbol{r}_{2} \frac{w_{k}\left(\boldsymbol{r}_{1}\right) w_{l}\left(\boldsymbol{r}_{2}\right)}{w_{k}\left(\boldsymbol{r}_{1}\right)+w_{l}\left(\boldsymbol{r}_{2}\right)}
$$

The cut-off radius $r_{c}=(1.475-0.866 \ln S) S$ is chosen to correctly capture the limit of long-range perturbations in an electron gas $\$ 4$. The MLWFs, giving rise to densities $\rho=w^{2}$, are assumed to be of the Slater form:

$$
w_{n}\left(\left\|\boldsymbol{r}-\boldsymbol{r}_{n}\right\|\right)=\frac{3^{3 / 4}}{\sqrt{\pi} S_{n}^{3 / 2}} e^{-\left(\sqrt{3} / S_{n}\right)\left\|\boldsymbol{r}-\boldsymbol{r}_{n}\right\|}
$$

characterized solely by their spread $S_{n}=\left\langle w_{n}\left|r^{2}\right| w_{n}\right\rangle-\left\langle w_{n}|r| w_{n}\right\rangle^{2}$ and center $r_{n}$.

We have previously shown that the dispersion interaction between two ensembles of charge density fragments can be obtained from the averaged sum over pair interactions of MLWFs 37 . Assuming an isotropic distribution of MLWF centers around the nuclei $I$ and $J$, at fixed distances, leads (to second leading-order) to $V_{\text {disp }}=-\sum_{n=6,8} C_{n}^{I J} / r_{I J}^{n}$, where the dispersion 
coefficients are:

$$
\begin{aligned}
& C_{6}^{I J}=\sum_{k \in I, l \in J} C_{6}^{k l} \\
& C_{8}^{I J}=\sum_{k \in I, l \in J} 5\left(d_{k}^{2}+d_{l}^{2}\right) C_{6}^{k l}
\end{aligned}
$$

where $d_{k, l}$ are the distances of the MLWF centers to their respective nuclei and $C_{6}^{k l}$ is computed for each pair of MLWFs according to Eq. 12. The determination of the parameter $b_{D}$ in Eq. 4, for the short-range damping of the dispersion interaction, is detailed below.

\section{Dipole- and force-fitting}

Not all parameters of the force field can be derived systematically from the electronic density. However, they can be determined numerically so as to best reproduce the atomic properties calculted by DFT: the total dipoles (permanent plus induced) of ions and molecules and the forces acting on them.

\section{Damping of charge-dipole interaction}

The first step in our parametrization procedure is to determine the parameters involved in Eq. 7 for the short-range damping of the charge-dipole interaction. This is achieved by numerically adjusting these parameters so as to minimize the error on the dipoles calculated using the classical force field, relative to the DFT ones on a number of representative configurations:

$$
\chi_{\mu}^{2}=\frac{1}{N_{\text {conf }}} \frac{1}{N_{\text {atom }}} \sum_{\text {conf }} \sum_{\text {atom }} \frac{\left\|\boldsymbol{\mu}^{\text {classical }}-\boldsymbol{\mu}^{D F T}\right\|^{2}}{\left\|\boldsymbol{\mu}^{D F T}\right\|^{2}}
$$

Together with the polarizabilities, these parameters complete the description of the polarization potential $V_{\text {pol }}$.

\section{Repulsion}

The parameters of the repulsive potential $V_{\text {rep }}$ in Eq. 5 can then be obtained by a similar procedure as the one used for the dipoles, if the functional used for the DFT calculation 
does not include dispersion interactions (e.g. PBE or BLYP) $\underline{46}, \underline{47}$ :

$$
\chi_{F}^{2}=\frac{1}{N_{\text {conf }}} \frac{1}{N_{\text {atom }}} \sum_{\text {conf }} \sum_{\text {atom }} \frac{\left\|\boldsymbol{F}^{\text {classical }}-\boldsymbol{F}^{D F T}\right\|^{2}}{\left\|\boldsymbol{F}^{D F T}\right\|^{2}}
$$

By ajusting the parameters for the damping of the charge-dipole interaction and for the repulsion on different physical quantities (dipoles and forces, respectively), we limit the risk of having a compensation of errors between the different terms of the potential.

\section{Further considerations}

The water-ion interactions are parametrized by applying the procedure described above on configurations of a system containing a single ion in bulk water. For the ion-ion interactions, we use configurations of the experimentally stable crystal phase under normal conditions: $\mathrm{NaCl}$ structure for $\mathrm{Li}^{+}, \mathrm{Na}^{+}, \mathrm{K}^{+}$and $\mathrm{Rb}^{+}, \mathrm{CsCl}$ structure for $\mathrm{Cs}^{+}, \mathrm{MgCl}_{2}$ structure for $\mathrm{Mg}^{2+}$, and $\mathrm{CaCl}_{2}$ structure for $\mathrm{Ca}^{2+}$ and $\mathrm{Sr}^{2+}$. The $\mathrm{Cl}-\mathrm{Cl}$ interactions must be the same among the different crystals in order to ensure the consistency and transferability of our potentials. The parameters for the $\mathrm{Cl}-\mathrm{Cl}$ repulsion are obtained for $\mathrm{LiCl}$, in which they are the most prominent, and the corresponding values are then used for all crystals. The $C_{6}$ and $C_{8}$ parameters for the $\mathrm{Cl}-\mathrm{Cl}$ dispersion interaction are obtained by averaging the values for the different crystals.

For the cation-anion repulsion (see Eq. 5 ), the force-fitting procedure results in $B$ parameters that are very close to each other among the alkaline ions on the one hand, and among the alkaline earth ions on the other hand. For the sake of simplicity, we use only one value for this parameter for each ion series. The $A$ parameters for the cation-anion repulsion are then readjusted to minimize Eq. 17 while keeping the $B$ value fixed. The final values for $A$ and the corresponding $\chi_{F}^{2}$ were practically unchanged by this constraint, thus confirming the relevance of this choice.

In order to further decrease the number of free parameter, the range of the short-range damping used for the cation-anion dispersion $b_{D}^{I J}$ (see Eqs. 3] and 4) is taken in most cases equal to that of the short-range repulsion $B^{I J}$. This assumption is not new $\underline{48}$, and it is justified by the notion that the long-range scaling of dispersion breaks down as the electronic fragments start overlapping, when the short-range repulsion comes into play. The damping of the $\mathrm{Cl}-\mathrm{Cl}$ dispersion is adjusted numerically so as to reproduce simultaneously the 
experimental density of all crystals. For the largest cations, $\mathrm{Cs}^{+}$and $\mathrm{Sr}^{2+}$, a value slightly smaller than $B^{\text {cation-Cl}}$ was needed to reproduce the experimental densities. Compared to the usual procedure of parametrizing a PIM from ab-initio simulations $\underline{11}, 34$, the systematic determination of the $C_{6}$ and $C_{8}$ coefficients and the assumption that $b_{D}=B$ dramatically reduce the number of parameters that need to be adjusted in order to reproduce the whole set of experimental densities. The damping parameter of the monovalent cation-water dispersion interaction was chosen equal to that for the corresponding monovalent cation- $\mathrm{Cl}^{-}$ dispersion interaction $b_{D}^{\text {ion-O }}=b_{D}^{\text {ion-Cl}}$, since the water molecules and the $\mathrm{Cl}^{-}$ions have approximately the same size. As far as the divalent cations are concerned, the attractive force arising at short distances from dispersion is negligible compared to the charge-charge and charge-dipole interactions. We can thus omit damping this interaction without any loss of accuracy. Similarly, the dispersion interaction between $\mathrm{Cl}^{-}$and water oxygen atom is not damped.

Overall, these considerations reduce the number of parameters for the interaction of all ions with water and of cations with chloride from 241 to 187, after the neglect of some terms for the reasons explained above, and to 170 by further assuming that the ranges of some interactions are equal. Out these 170, only 82 are adjusted numerically from the dipoleand force-matching procedures of section IIC, while the rest are computed as expained in section $1 \mathrm{~B}$.

\section{E. Simulation details}

The parametrization of the force field from ab-initio simulations is achieved using representative configurations of the aqueous ions and the ionic crystals. For each ionic species, $\sim 100$ configurations of a system containing a single ion and 32 water molecules are generated using the force-field of Dang et al. $\underline{\underline{49}} \underline{\underline{51}}$ for the monovalent ions, and that of Yu et al. $\underline{52}$ for the divalent ions. DFT calculations were then performed on these configurations with the BLYP functional ${ }^{53,54}$ (exept for the $\mathrm{Rb}^{+}$, for which the PBE functional ${ }^{55}$ was used). The Troullier-Martins $\frac{56}{6}\left(\mathrm{Cl}^{-}, \mathrm{Cs}^{+}\right.$and $\left.\mathrm{K}^{+}\right)$and Goedecker-Teter-Hutter $\underline{57-59}\left(\mathrm{Na}^{+}, \mathrm{Rb}^{+}, \mathrm{Mg}^{2+}\right.$, $\mathrm{Ca}^{2+}$ and $\mathrm{Sr}^{2+}$ ) pseudopotentials were used, with a plane-wave basis set and an energy cutoff of at least $70 \mathrm{Ry}$. Similarly, configurations of crystals containing between 16 and $108 \mathrm{MCl}$ or $\mathrm{MCl}_{2}$ units, are used to perform the DFT calculations, with the same functionals and 
pseudopotentials as for the ions in water. In each case, after determining the electronic density, the forces acting on each atom are computed and the dipoles are calculated from the MLWFs as described above. The $C_{6}$ and $C_{8}$ dispersion parameters are computed from the spreads and distances to the center of the MLWFs, which result from the localization procedure. The polarizabilities are calculated as explained above, by applying an external field using the Berry phase representation ${ }^{42}$. All ab-initio calculations were performed using the CPMD simulation package ${ }^{60}$ (exept for those involving $\mathrm{Ca}^{2+}$, performed with $\mathrm{CP} 2 \mathrm{~K}$ simulation package $\left.e^{61}\right)$, while classical forces and dipoles are computed on the same configurations with FIST, the classical MD module of the CP2K simulation package $\frac{61}{}$. The numerical minimization of Eqs. (16) and (17) is performed using the Minuit library $\underline{62}$.

\section{F. Parametrization: Results}

The computed polarizabilities for all the ions are summarized in table【, As expected, the polarizability increases when going down along columns of the periodic table (alkaline and alkaline earths series), while a decrease is observed when going from left to right along rows $\left(\mathrm{Na}^{+}\right.$to $\mathrm{Mg}^{2+}, \mathrm{K}^{+}$to $\mathrm{Ca}^{2+}$ and $\mathrm{Rb}^{+}$to $\left.\mathrm{Sr}^{2+}\right)$. For cations, the condensed phase polarizability is comparable to that in the gas phase, except for $\mathrm{Cs}^{+}$. For the chloride anion, however, the confinement of electrons by the surrounding water molecules results in a significant decrease of the polarizability (approximately 35\%). A more detailed discussion has been given in Ref. .42 .

Interestingly, as indicated in Table II, the (induced) dipole moment of cations is always very small compared to that of the chloride anion and water. This can be explained by the combination of two factors. First, most cations have a small $\left(\mathrm{K}^{+}, \mathrm{Sr}^{2+}\right)$ or very small $\left(\mathrm{Li}^{+}\right.$, $\left.\mathrm{Na}^{+}, \mathrm{Mg}^{2+}, \mathrm{Ca}^{2+}\right)$ polarizability. Second, all cations have a highly symmetric hydration sphere, which results in very weak local electric fields to polarize them. Because the induced dipoles are very small, they are not easily reproduced by the classical force field (typical errors are of the order of $100 \%$ ), but they do not contribute significantly to the polarization energy $V_{\text {pol }}$, which is dominated by the interaction of the ionic charge with the dipole of water, and hence to the forces. For the sake of simplicity, we thus decided to neglect the polarizability of all cations and not include any additional degrees of freedom to describe their induced dipoles. 


\begin{tabular}{|l|c|c|}
\hline Ion & $\alpha\left(\AA^{3}\right)$ & $\sqrt{\left\langle\boldsymbol{\mu}^{2}\right\rangle}$ (Debye) \\
\hline \hline $\mathrm{Li}^{+}$ & 0.03 & 0.002 \\
\hline $\mathrm{Na}^{+}$ & 0.18 & 0.014 \\
\hline $\mathrm{K}^{+}$ & 0.81 & 0.062 \\
\hline $\mathrm{Rb}^{+}$ & 1.32 & 0.097 \\
\hline $\mathrm{Cs}^{+}$ & 2.02 & 0.153 \\
\hline \hline $\mathrm{Mg}^{2+}$ & 0.08 & 0.010 \\
\hline $\mathrm{Ca}^{2+}$ & 0.44 & 0.026 \\
\hline $\mathrm{Sr}^{2+}$ & 0.81 & 0.071 \\
\hline \hline $\mathrm{Cl}^{-}$ & 3.50 & 0.415 \\
\hline
\end{tabular}

TABLE II: Polarizability $\alpha$ and magnitude of the induced dipole of each ion $\sqrt{\left\langle\boldsymbol{\mu}^{2}\right\rangle}$. The latter is $1.18 \mathrm{D}$ for water.

The parameters for the cation-water interaction are summarized in Table III, and those for the chloride-water interaction are given in Table IV] Finally, all parameters for the ionion interactions are given in Table $\mathrm{V}$. The resulting repulsion potentials $V_{\text {rep }}$ between water and the various cations, plotted in Fig. 2, nicely reflect the expected increase in ionic size along the alkaline and alkaline earth series. Furthermore, a comparable repulsion is observed for isoelectronic species such as $\mathrm{Na}^{+}$and $\mathrm{Mg}^{2+}, \mathrm{K}^{+}$and $\mathrm{Ca}^{2+}$, and $\mathrm{Rb}^{+}$and $\mathrm{Sr}^{2+}$. In line with the polarizabilities, the $C_{6}$ and $C_{8}$ dispersion coefficients for the ion-water interaction increase along the alkaline and alkaline earths series, while a decrease is observed from left to right along rows of the periodic table. The same trends hold for the repulsion and dispersion interactions between the cations and the chloride anion.

We now examine the performance of the force field in terms of reproducing the $a b$ initio dipoles and forces. Figure 3 illustrates the comparison between the forces on the ion calculated with the classical force field (without dispersion) and those obtained from the DFT calculations, for the $\mathrm{Ca}^{2+}$ cation. From table VI, the relative error of the force, $\sqrt{\chi_{F}^{2}}$, on the $\mathrm{Ca}^{2+}$ ion, with respect to the DFT result, is approximately $23 \%$. This can be considered as a good match, especially when comparing to the corresponding results obtained by using the Dang potential $\underline{63}$ (with the same water model), which results in a 


\begin{tabular}{|l|c|c|c|c|c|c|c|}
\hline System & $\mathrm{A}^{\text {ion-O }}(\mathrm{Ha})$ & $\mathrm{B}^{\text {ion-O }}\left(\AA^{-1}\right)$ & $\mathrm{C}_{6}^{\text {ion-O }}\left(\mathrm{Ha} . \AA^{6}\right)$ & $\mathrm{C}_{8}^{\text {ion-O }}\left(\mathrm{Ha} . \AA^{8}\right)$ & $\mathrm{b}_{D}^{I J}\left(\AA^{-1}\right)$ & $\mathrm{b}^{\text {ion-M }}\left(\AA^{-1}\right)$ & $\mathrm{c}^{\text {ion-M }}$ \\
\hline \hline $\mathrm{Li}^{+}$-water & 24.75 & 4.094 & $1.103 \times 10^{-2}$ & $1.037 \times 10^{-2}$ & 3.000 & 4.011 & 2.950 \\
\hline $\mathrm{Na}^{+}$-water & 711.1 & 5.061 & $1.335 \times 10^{-1}$ & $1.572 \times 10^{-1}$ & 3.000 & 1.562 & $6.839 \times 10^{-1}$ \\
\hline $\mathrm{K}^{+}$-water & 125.7 & 3.735 & $7.530 \times 10^{-1}$ & 1.206 & 3.000 & 1.315 & $4.623 \times 10^{-1}$ \\
\hline $\mathrm{Rb}^{+}$-water & 157.8 & 3.656 & 1.225 & 2.267 & 3.000 & 1.248 & $4.765 \times 10^{-1}$ \\
\hline $\mathrm{Cs}^{+}$-water & 269.4 & 3.635 & 2.040 & 4.644 & 1.800 & 2.524 & 2.948 \\
\hline \hline $\mathrm{Mg}^{2+}$-water & 65.67 & 3.963 & $6.408 \times 10^{-2}$ & $7.23 \times 10^{-3}$ & - & 3.963 & 2.820 \\
\hline $\mathrm{Ca}^{2+}$-water & 57.94 & 3.327 & $5.055 \times 10^{-1}$ & $7.502 \times 10^{-1}$ & - & 3.327 & 3.000 \\
\hline $\mathrm{Sr}^{2+}$-water & 41.55 & 2.991 & $9.159 \times 10^{-1}$ & 1.576 & - & 2.991 & 2.041 \\
\hline
\end{tabular}

TABLE III: Parameters for the cation-water interactions. As for water-water interactions, respulsion and dispersion involve the oxygen atom, while electrostatic interactions involve the additional $\mathrm{M}$ site. The damping parameter $b_{D}$ for the dispersion interaction for the monovalent ions is chosen equal to that of the corresponding cation-chloride interaction (see text and table $\mathrm{V}$ ). The electrostatic damping is between the water dipole and cation charge.

\begin{tabular}{|l|c|c|c|c|c|c|c|c|}
\hline System & $\mathrm{A}^{\text {ion-O }}(\mathrm{Ha})$ & $\mathrm{B}^{\text {ion-O }}\left(\AA^{-1}\right)$ & $\mathrm{C}_{6}^{\text {ion-O }}\left(\mathrm{Ha} . \AA^{6}\right)$ & $\mathrm{C}_{8}^{\text {ion-O }}\left(\mathrm{Ha} . \AA^{8}\right)$ & $\mathrm{b}^{\text {ion-H }}\left(\AA^{-1}\right)$ & $\mathrm{c}^{\text {ion-H }}$ & $\mathrm{b}^{\text {ion-M }}\left(\AA^{-1}\right)$ & $\mathrm{c}^{\text {ion-M }}$ \\
\hline \hline Cl-water & 499.63 & 3.560 & 2.039 & 4.296 & 4.794 & 1.093 & 2.444 & -1.901 \\
\hline
\end{tabular}

TABLE IV: Parameters for the chloride-water interactions. The dipole damping is between the $\mathrm{Cl}^{-}$ and the water charges. For the reasons already explained, there is no damping of the dispersion.

relative error of approximately 320\%. Similarly, our results for $\mathrm{Sr}^{2+}$ show a relative error of $36 \%$, compared to $501 \%$ with the force field from the literature $\frac{64}{4}, 49 \%$ vs. $66 \%$ for $\mathrm{Na}^{+}$, $48 \%$ vs. $131 \%$ for $\mathrm{Cs}^{+}$and $53 \%$ vs. $104 \%$ for $\mathrm{Cl}^{-}$. Overall, the forces on all ions are well reproduced by the present force field. The largest contributions to the relative error (see Eq. 17) correspond to the smaller forces.

Table VII reports the $\chi^{2}$ values obtained on the crystals for the forces on both cations and anions, as well as for the dipole of the anions. Comparison with Table VI indicates that a similar accuracy is obtained for both the crystals and the ions in solution, suggesting that the force field should perform well under both conditions. This result is also encouraging from the point of view of the transferability and the possible prediction of the solubility of 


\begin{tabular}{|c|c|c|c|c|c|c|c|c|}
\hline System & Ion pair IJ & $\mathrm{A}^{I J}(\mathrm{Ha})$ & $\mathrm{B}^{I J}\left(\AA^{-1}\right)$ & $\mathrm{C}_{6}^{I J}\left(\mathrm{Ha} . \AA^{6}\right)$ & $\mathrm{C}_{8}^{I J}\left(\mathrm{Ha} . \AA^{8}\right)$ & $\mathrm{b}_{D}^{I J}\left(\AA^{-1}\right)$ & $\mathrm{b}^{I J}\left(\AA^{-1}\right)$ & $\mathrm{c}^{I J}$ \\
\hline \multirow[t]{3}{*}{$\mathrm{LiCl}$} & $\mathrm{Li}^{+}-\mathrm{Li}^{+}$ & 481.9 & 6.958 & $2.727 \times 10^{-4}$ & $5.570 \times 10^{-10}$ & 6.958 & - & - \\
\hline & $\mathrm{Li}^{+}-\mathrm{Cl}^{-}$ & 15.56 & 3.000 & $2.369 \times 10^{-2}$ & $2.511 \times 10^{-2}$ & 3.000 & 3.128 & 1.433 \\
\hline & $\mathrm{Cl}^{-}{ }_{-} \mathrm{Cl}^{-}$ & 698.4 & 3.777 & 5.951 & 12.85 & 1.650 & - & - \\
\hline \multirow[t]{3}{*}{$\mathrm{NaCl}$} & $\mathrm{Na}^{+}-\mathrm{Na}^{+}$ & $1.701 \times 10^{-2}$ & 4.965 & $2.914 \times 10^{-2}$ & $1.394 \times 10^{-2}$ & 4.965 & - & - \\
\hline & $\mathrm{Na}^{+}-\mathrm{Cl}^{-}$ & 44.43 & 3.000 & $2.971 \times 10^{-1}$ & $3.785 \times 10^{-1}$ & 3.000 & 2.775 & 2.040 \\
\hline & $\mathrm{Cl}^{-}-\mathrm{Cl}^{-}$ & 698.4 & 3.777 & 5.951 & 12.85 & 1.650 & - & - \\
\hline \multirow[t]{3}{*}{$\mathrm{KCl}$} & $\mathrm{K}^{+}-\mathrm{K}^{+}$ & 174.9 & 5.000 & $7.172 \times 10^{-1}$ & $9.260 \times 10^{-1}$ & 5.000 & - & - \\
\hline & $\mathrm{K}^{+}-\mathrm{Cl}^{-}$ & 82.92 & 3.000 & 1.973 & 3.347 & 3.000 & 1.282 & $9.059 \times 10^{-1}$ \\
\hline & $\mathrm{Cl}^{-}-\mathrm{Cl}^{-}$ & 698.4 & 3.777 & 5.951 & 12.85 & 1.650 & - & - \\
\hline \multirow[t]{3}{*}{$\mathrm{RbCl}$} & $\mathrm{Rb}^{+}-\mathrm{Rb}^{+}$ & $1.235 \times 10^{-2}$ & 3.485 & 2.235 & 3.908 & 3.485 & - & - \\
\hline & $\mathrm{Rb}^{+}{ }_{-} \mathrm{Cl}^{-}$ & 108.0 & 3.000 & 3.755 & 7.223 & 3.000 & 1.460 & $9.825 \times 10^{-1}$ \\
\hline & $\mathrm{Cl}^{-}-\mathrm{Cl}^{-}$ & 698.4 & 3.777 & 5.951 & 12.85 & 1.650 & - & - \\
\hline \multirow[t]{3}{*}{$\mathrm{CsCl}$} & $\mathrm{Cs}^{+}{ }_{-} \mathrm{Cs}^{+}$ & 353.0 & 3.782 & 7.325 & 18.64 & 3.782 & - & - \\
\hline & $\mathrm{Cs}^{+}-\mathrm{Cl}^{-}$ & 150.1 & 3.000 & 7.339 & 16.96 & 1.800 & 1.541 & $4.665 \times 10^{-1}$ \\
\hline & $\mathrm{Cl}^{-}-\mathrm{Cl}^{-}$ & 698.4 & 3.777 & 5.951 & 12.85 & 1.650 & - & - \\
\hline \multirow[t]{3}{*}{$\mathrm{MgCl}_{2}$} & $\mathrm{Mg}^{+}-\mathrm{Mg}^{2+}$ & $2.231 \times 10^{-1}$ & 4.995 & $1.095 \times 10^{-2}$ & $4.066 \times 10^{-3}$ & 4.995 & - & - \\
\hline & $\mathrm{Mg}^{2+}{ }_{-} \mathrm{Cl}^{-}$ & 85.84 & 3.400 & $1.471 \times 10^{-1}$ & $2.102 \times 10^{-1}$ & 3.400 & 2.886 & 2.113 \\
\hline & $\mathrm{Cl}^{-}-\mathrm{Cl}^{-}$ & 698.4 & 3.777 & 5.951 & 12.85 & 1.650 & - & - \\
\hline \multirow[t]{3}{*}{$\mathrm{CaCl}_{2}$} & $\mathrm{Ca}^{2+}-\mathrm{Ca}^{2+}$ & $1.289 \times 10^{-1}$ & 3.941 & $3.274 \times 10^{-1}$ & $3.456 \times 10^{-1}$ & 3.941 & - & - \\
\hline & $\mathrm{Ca}^{2+}{ }_{-} \mathrm{Cl}^{-}$ & 236.3 & 3.400 & 1.168 & 1.883 & 3.400 & 2.052 & 1.268 \\
\hline & $\mathrm{Cl}^{-}-\mathrm{Cl}^{-}$ & 698.4 & 3.777 & 5.951 & 12.85 & 1.650 & - & - \\
\hline \multirow[t]{3}{*}{$\mathrm{SrCl}_{2}$} & $\mathrm{Sr}^{2+}-\mathrm{Sr}^{2+}$ & 5.513 & 4.735 & 1.259 & 1.867 & 4.735 & - & - \\
\hline & $\mathrm{Sr}^{2+}{ }_{-} \mathrm{Cl}^{-}$ & 269.4 & 3.400 & 2.697 & 5.066 & 2.400 & 3.103 & 2.939 \\
\hline & $\mathrm{Cl}^{-}-\mathrm{Cl}^{-}$ & 698.4 & 3.777 & 5.951 & 12.85 & 1.650 & - & - \\
\hline
\end{tabular}

TABLE V: Parameters for the ion-ion interactions.

these crystals. Comparison between tables $\mathrm{VIT}$ and VIII illustrates the better performance of the present model compared to those of Dang and coworkers $\underline{49}-\underline{51}$.

Neglecting the polarizability of cations does not prevent us from obtaining a good de- 

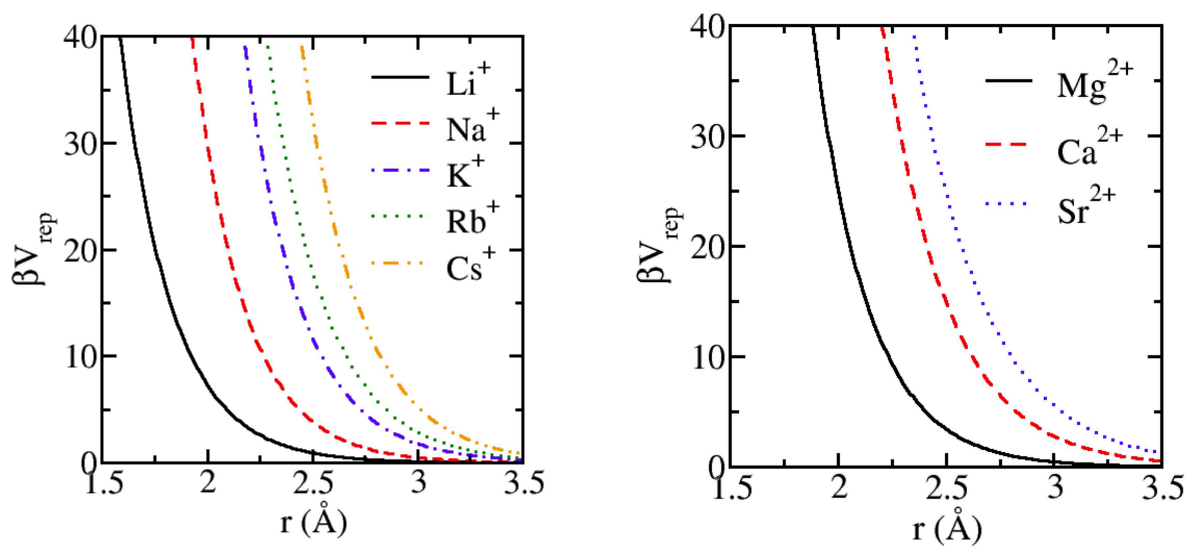

FIG. 2: Repulsion potential between water and alkaline cations (a) and alkaline earth cations (b), in units of the thermal energy $\beta^{-1}=k_{B} T$.

scription of the forces acting on them, as can be seen in table VI. These forces are even better described than those on the chloride ion, whose polarizability is explicitly taken into account. Nevertheless, for the reasons mentioned in the introduction, it is essential to correctly reproduce the polarization of water molecules around ions. Table VI also indicates the relative error on the dipole of water molecules in the first solvation shell of the ions. The combination of the Dang-Chang water model with the present model for the ion-water interactions provides a very good description of the polarization of water, with relative errors between 5 and $10 \%$ for all ions except $\mathrm{Mg}^{2+}(13 \%)$.

\section{VALIDATION}

Having shown that our force field is able to correctly reproduce the ab-initio dipoles and forces, we now turn to its validation against experimental data pertaining to the structure, thermodynamics and dynamics of aqueous ions at infinite dilution, as well as to the density of ionic crystals. We finally investigate the transferability of the force field to concentrated solutions, which where not taken into account when "designing" the force field. It is worth 


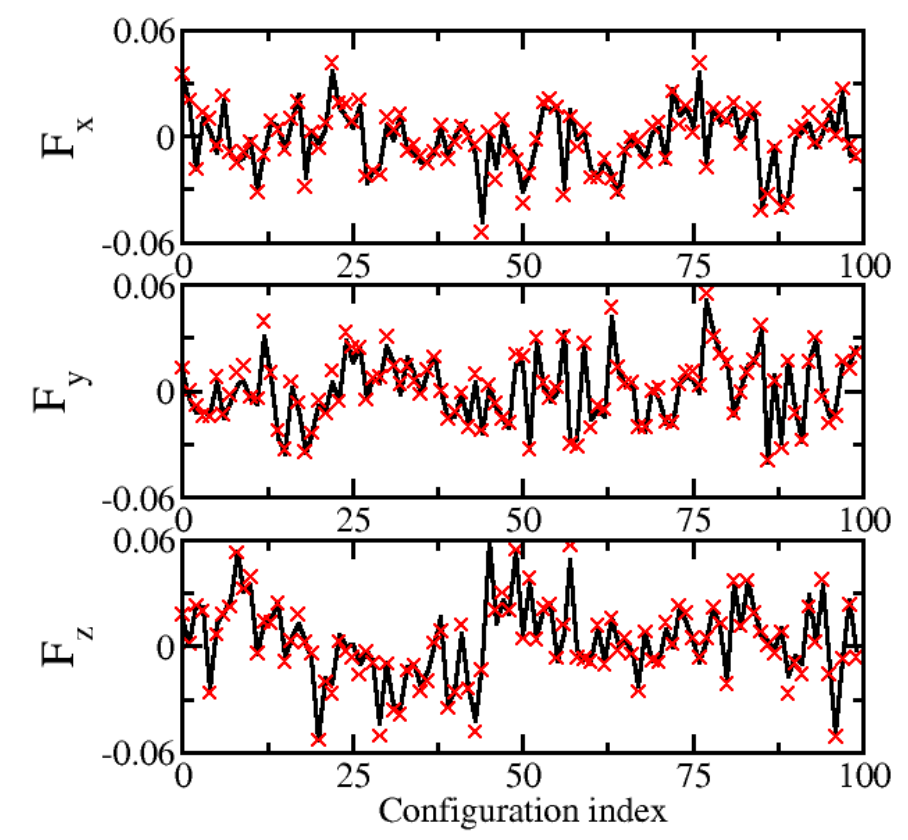

FIG. 3: Force (in atomic units) acting on the $\mathrm{Ca}^{2+}$ ion. The prediction of the classical force field (lines) for the 3 components $F_{x}, F_{y}$ and $F_{z}$ are compared to the DFT result $(\times)$, for 100 configurations composed of 32 water molecules and $1 \mathrm{Ca}^{2+}$.

pointing out here that we use experimental data only for validation purposes, in contrast with all other force fields for aqueous ions, which use some experimental data for calibration of the parameters. Out of the 241 parameters defining the force field for the present set of ions, only 3 (the dispersion damping parameters $b_{D}^{\mathrm{Cl}-\mathrm{Cl}}, b_{D}^{\mathrm{Cs}-\mathrm{Cl}}$ and $b_{D}^{\mathrm{Sr}-\mathrm{Cl}}$ ) are determined with the use of experimental data, namely the densities of the 8 crystals. In particular, no experimental data on aqueous ions is used during the calibration process.

\section{A. Simulation details}

For ions at infinite dilution, the system contains a single ion and 215 water molecules in a cubic box of size $L=18.65 \AA$. For the crystals, the systems consist of $256 \mathrm{LiCl}, \mathrm{NaCl}, \mathrm{KCl}$ or $\mathrm{RbCl}, 342 \mathrm{CsCl}, 192 \mathrm{MgCl}_{2}$ or $\mathrm{CaCl}_{2}$, or $256 \mathrm{SrCl}_{2}$. Systems for concentrated solutions are composed of $27 \mathrm{NaCl}, \mathrm{KCl}$ and 458 water molecules in cubic box of sizes $24.4167 \AA$ and $24.638 \AA$, respectively. Electrostatic interactions are computed using a dipolar Ewald sum ${ }^{65,66}$, with a tolerance of $1.10^{-7}$ to obtain the self-consistent dipole moments. Molecular 


\begin{tabular}{|l|c|c|c|}
\hline Ion & $\chi_{\text {F-ion }}^{2}$ & $\chi_{\mu-H_{2} O}^{2}$ & $\chi_{\mu \text {-ion }}^{2}$ \\
\hline \hline $\mathrm{Li}^{+}$ & $1.56 \times 10^{-1}$ & $1.76 \times 10^{-3}$ & - \\
\hline $\mathrm{Na}^{+}$ & $2.36 \times 10^{-1}$ & $7.81 \times 10^{-3}$ & - \\
\hline $\mathrm{K}^{+}$ & $1.11 \times 10^{-1}$ & $2.79 \times 10^{-3}$ & - \\
\hline $\mathrm{Rb}^{+}$ & $1.02 \times 10^{-1}$ & $2.35 \times 10^{-3}$ & - \\
\hline $\mathrm{Cs}^{+}$ & $2.28 \times 10^{-1}$ & $3.08 \times 10^{-3}$ & - \\
\hline \hline $\mathrm{Mg}^{2+}$ & $9.97 \times 10^{-2}$ & $1.78 \times 10^{-2}$ & - \\
\hline $\mathrm{Ca}^{2+}$ & $5.35 \times 10^{-2}$ & $9.05 \times 10^{-3}$ & - \\
\hline $\mathrm{Sr}^{2+}$ & $1.27 \times 10^{-1}$ & $2.73 \times 10^{-3}$ & - \\
\hline \hline $\mathrm{Cl}^{-}$ & $2.84 \times 10^{-1}$ & $3.40 \times 10^{-3}$ & $2.05 \times 10^{-1}$ \\
\hline
\end{tabular}

TABLE VI: $\chi^{2}$ for the forces on the ions and the dipoles of water and the ions.

\begin{tabular}{|l|c|c|c|}
\hline Crystal & $\chi_{\mathrm{F}-\mathrm{M}^{x+}}^{2}$ & $\chi_{\mathrm{F}-\mathrm{Cl}^{-}}^{2}$ & $\chi_{\mu-\mathrm{Cl}^{-}}^{2}$ \\
\hline \hline $\mathrm{LiCl}$ & $1.13 \times 10^{-1}$ & $1.74 \times 10^{-2}$ & $1.91 \times 10^{-1}$ \\
\hline $\mathrm{NaCl}$ & $2.52 \times 10^{-2}$ & $1.12 \times 10^{-2}$ & $1.87 \times 10^{-1}$ \\
\hline $\mathrm{KCl}$ & $7.88 \times 10^{-2}$ & $5.61 \times 10^{-2}$ & $7.28 \times 10^{-1}$ \\
\hline $\mathrm{RbCl}$ & $4.77 \times 10^{-2}$ & $6.46 \times 10^{-2}$ & $6.66 \times 10^{-1}$ \\
\hline $\mathrm{CsCl}$ & $2.23 \times 10^{-2}$ & $1.15 \times 10^{-1}$ & $4.30 \times 10^{-1}$ \\
\hline \hline $\mathrm{MgCl}_{2}$ & $2.62 \times 10^{-1}$ & $8.45 \times 10^{-2}$ & $1.90 \times 10^{-2}$ \\
\hline $\mathrm{CaCl}_{2}$ & $5.58 \times 10^{-2}$ & $8.46 \times 10^{-3}$ & $2.27 \times 10^{-1}$ \\
\hline $\mathrm{SrCl}_{2}$ & $3.61 \times 10^{-2}$ & $3.95 \times 10^{-2}$ & $5.69 \times 10^{-2}$ \\
\hline
\end{tabular}

TABLE VII: $\chi^{2}$ in crystals, for the forces on the cations and anions, and the dipoles of anions.

dynamics in the canonical ensemble are performed using a Nose-Hoover thermostat with a time constant of 1 ps. The system is first equilibrated for $250 \mathrm{ps}$, and the properties are determined from subsequent $2.75 \mathrm{~ns}$ runs. The density of the crystals is determined from simulations in the NPT ensemble at $P=1$ bar. The thermostat is the same than the one used for the NVT ensemble and the barostat is an extension of the one by Martyna et al. $\underline{67}$. All simulations are performed using the CP2K simulation package ${ }^{61}$. 


\begin{tabular}{|l|c|c|}
\hline Crystal & $\chi_{\mathrm{F}_{-} \mathrm{M}^{x+}}^{2}$ & $\chi_{\mathrm{F}-\mathrm{Cl}^{-}}^{2}$ \\
\hline $\mathrm{LiCl}$ & 3.51 & 28.0 \\
\hline $\mathrm{NaCl}$ & $4.46 \times 10^{-1}$ & 3.50 \\
\hline $\mathrm{KCl}$ & 4.38 & 4.10 \\
\hline $\mathrm{CsCl}$ & 2.24 & 2.56 \\
\hline \hline $\mathrm{CaCl}_{2}$ & 1.03 & $9.85 \times 10^{-1}$ \\
\hline $\mathrm{SrCl}_{2}$ & 5.47 & 8.28 \\
\hline
\end{tabular}

TABLE VIII: $\chi^{2}$ in crystals, for the forces on the cations and anions, with the polarizable DangChang models.

\section{B. Solvation of ions: structure}

We first inverstigate the structure of the solvation shells around ions by computing radial distribution functions, reported for the cations in Fig. 4. As usually observed, the position of the first maximum gradually shifts towards larger distances when switching from $\mathrm{Li}^{+}$to $\mathrm{Cs}^{+}$ and from $\mathrm{Mg}^{2+}$ to $\mathrm{Sr}^{2+}$, while the value of the maximum decreases and the peak broadens. On the contrary, moving right along the rows of the periodic table results in a closer and sharper peak. This arises from the stronger electrostatic interaction, since the ion-water repulsion remains comparable, as discussed previously, and reflects a tighter first solvation shell.
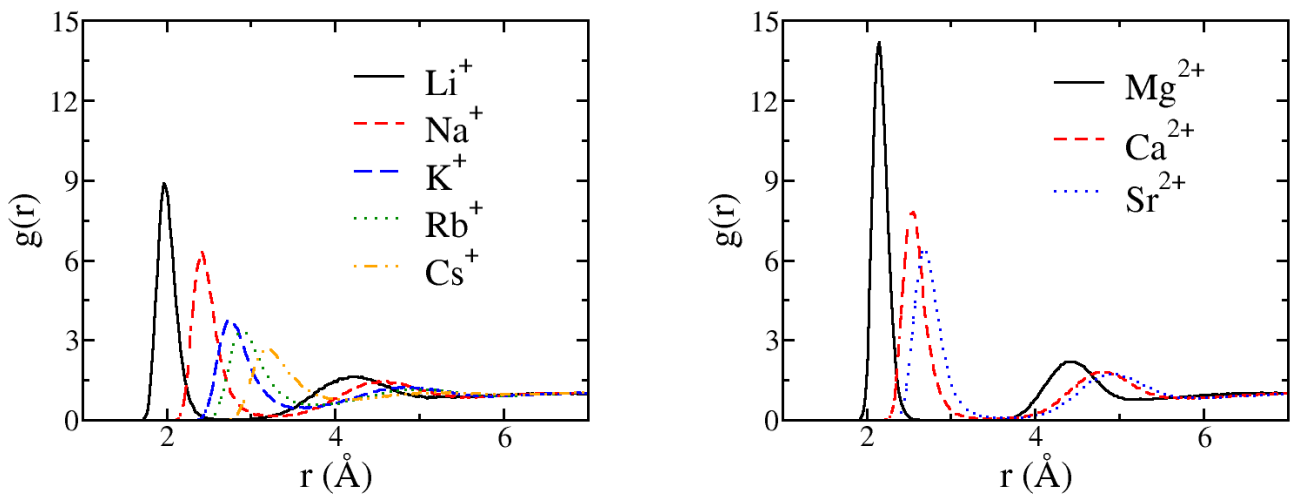

FIG. 4: Ion-oxygen radial distribution functions for the aqueous cations. 
The positions of the first maximum and the coordination numbers, defined as the integral of the ion- $\mathrm{O}$ (water) radial distribution function from the origin out to the first minimum, are summarized in Table $\mathrm{IX}$, together with the corresponding experimental values. The value and error estimates of the coordination numbers are determined from the plateau of the running values. Remarkably, all simulated data fall in the reported experimental ranges. Particularly encouraging is the agreement with experimental data for the three divalent ions. While several force fields are able to correctly predict the position and number of neighbours for the $\mathrm{Mg}^{2+}$ ion, many of them fail to correctly reproduce that of $\mathrm{Ca}^{2+}$. As an example, a force field by Yu et al. based on a Drude model of polarizability, which accurately describes the hydration free energies, predicts a coordination number of 6 for this ion $\underline{\underline{52}}$. Our result is very close to the value of 7.3 obtained with the popular AMOEBA force field used for biomolecular simulations ${ }^{68}$. The previously available model for $\mathrm{Ca}^{2+}$, with the present water model, predicts a distance of $2.45 \AA$, within the experimental range, but it used the EXAFS data of $2.43 \AA$ in the parametrization process. In the case of $\mathrm{Sr}^{2+}$, we find a distance very close to the anomalous X-ray diffraction value of $2.67 \AA^{\underline{69}}$ and a coordination number which is within the reported experimental range.

When comparing simulation results for an ion at infinite dilution with experiments, one should pay attention to the experimental conditions, in particular the concentration and the nature of the counterion. For example, Smirnov and Trostin reported an increase of the $\mathrm{Cs}^{+}$coordination number with decreasing concentration $\underline{\underline{70}}$. It is thus not surprising to find our result on the larger side of the experimental range. Moreover, results using $\mathrm{ClO}_{4}^{-}$as a counterion instead of $\mathrm{Cl}^{-}$are less likely to be polluted by the formation of ion pairs. The distances of 2.12 and $2.65 \AA$ between the cation and the nearest water oxygen, reported with $\mathrm{ClO}_{4}^{-}$in $\mathrm{Ref} . \underline{\underline{71}}$ for $\mathrm{Mg}^{2+}$ and $\mathrm{Sr}^{2+}$, respectively, are in very good agreement with ours (2.13 and 2.68).

Since most force fields include some experimental data on the structure during the calibration process $\frac{52,63.64}{6}$, it is possible to obtain a good agreement. When such data is not included as a target property, the predicted structure may not be very accurate. As an example, Horinek et al. parametrized a simple non-polarizable force field optimized for the simulation of solvation thermodynamics $\underline{72}$. The structural properties, used only for validation purposes, revealed a tendency to underestimate the distances to the nearest water molecules for cations. Our results for the chloride ion are very good, as they fall exactly on 
the EXAFS value of $2.11 \AA$ determined by Dang et al., whereas many force fields tend to predict too large a distance for the first peak ${ }^{63.72}$, even though they include such structural properties in the fitting procedure ${ }^{63}$.

\begin{tabular}{|l|c|c|c|c|}
\hline Ion & \multicolumn{2}{|c|}{ Position $(\AA)$} & \multicolumn{2}{l|}{ Coordination Number } \\
\hline & Sim & Exp & Sim & Exp \\
\hline \hline $\mathrm{Li}^{+}$ & 1.96 & $1.90-2.25$ & 4.0 & 4 \\
\hline $\mathrm{Na}^{+}$ & 2.41 & $2.41-2.50$ & $5.7 \pm 0.1$ & $4-8$ \\
\hline $\mathrm{K}^{+}$ & 2.74 & $2.60-2.92$ & $6.45 \pm 0.25$ & $4-8$ \\
\hline $\mathrm{Rb}^{+}$ & 2.88 & $2.80-3.05$ & $7.05 \pm 0.25$ & $6-8$ \\
\hline $\mathrm{Cs}^{+}$ & 3.20 & $2.95-3.21$ & $8.3 \pm 0.8$ & $6-8$ \\
\hline \hline $\mathrm{Mg}^{2+}$ & 2.13 & $2.00-2.15$ & 6.0 & 6 \\
\hline $\mathrm{Ca}^{2+}$ & 2.53 & $2.40-2.58$ & $7.24 \pm 0.02$ & $7-9$ \\
\hline $\mathrm{Sr}^{2+}$ & 2.68 & $2.57-2.67$ & $7.81 \pm 0.05$ & $7.3-10.3$ \\
\hline \hline $\mathrm{Cl}^{-}$ & 3.11 & $3.05-3.18$ & $6.12 \pm 0.12$ & $5.3-6.4$ \\
\hline
\end{tabular}

TABLE IX: Structural properties: position of the first maximum in radial distribution function and coordination number. The experimental values are taken from Refs, $\underline{63,69}, \underline{71}, 73$

Positions and coordination numbers cannot be measured directly, and the experimental values are the outcome of a complex numerical analysis of the raw data, which typically involves several Fourier transforms and filters which can influence the final result. A more stringent test of the force field thus consists in comparing the experimental signal to that obtained by computing the experimental observables on configurations generated by molecular simulation. An example of such a test is given in Fig. 5, which compares the experimental EXAFS signal for aqueous $\mathrm{Ca}^{2+}$, obtained from Ref. ${ }^{63}$, to that predicted from our configurations using the FEFF8 code, which uses an updated version of the Rehr et al. algorithm $\frac{74}{4}$ to evaluate multiple electron scattering series. The agreement is seen to be very good in the $k>3 \AA^{-1}$ part of the spectrum, both in terms of the amplitude (which reflect the number of neighbours) and the frequency of oscillations (related to their position). 


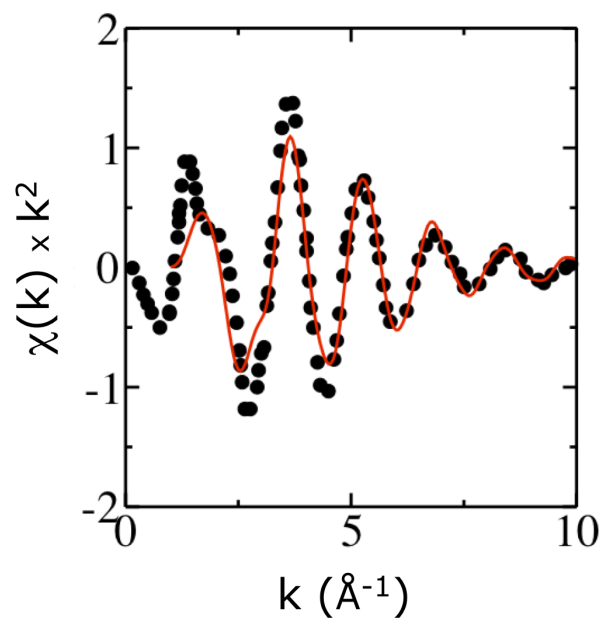

FIG. 5: Comparison between simulated and experimental EXAFS 63 signal for aqueous $\mathrm{Ca}^{2+}$. The agreement is very good both for the amplitude, which reflects the number of neighbours, and frequency of the oscillations, related to their position.

\section{Solvation of ions: hydration free energy}

Among all the ionic properties one aims to predict, the hydration free energy $\Delta G_{\text {hyd }}$ is probably the most important, since it relates to the ability of ions to accomodate their solvation shell when approaching an interface or other ions. This quantity is almost always one of the target properties used to design force fields. Whereas absolute values are difficult to determine, differences in hydration free energies can be easily computed using a thermodynamic integration procedure without worrying about the numerous corrections $\underline{75}, 76$ (for system size, boundary conditions, and the treatment of electrostatic interactions) needed for the former. The difference $\Delta \Delta G_{\text {hyd }} \approx \Delta \Delta F_{\text {hyd }}=\Delta F_{\text {hyd }}^{\mathrm{K}}-\Delta F_{\text {hyd }}^{\mathrm{Na}}$ can be determined from a thermodynamic path (transmutation) connecting the systems, by introducing a mixed Hamiltonian $H(\lambda)=\lambda H_{\mathrm{K}}+(1-\lambda) H_{\mathrm{Na}}$ for $\lambda \in[0,1]$, as

$$
\Delta \Delta F=\int_{0}^{1}\left\langle\frac{\partial H}{\partial \lambda}\right\rangle d \lambda
$$

For the monovalent ions, we use a 6-point Gaussian quadrature 77,78 to compute the integral, except for the $\mathrm{Li}^{+}-\mathrm{Cs}^{+}$transmutation, for which we use an 8-point quadrature. Details on this standard quadrature procedure can be found in Ref. $\underline{77}$. In the case of the divalent ions, where $\partial_{\lambda} H(\lambda)$ shows a linear variation in $\lambda$, a simpler trapezoidal rule can be used to 
approximate the integral. In this case we used ten equally spaced points (0.1) for $\lambda_{i}$ within the interval $[0,1]$.

\begin{tabular}{|l|c|c|}
\hline Transmutation & $\Delta \Delta G_{\text {hyd }}^{\text {sim }}(\mathrm{kcal} / \mathrm{mol})$ & $\Delta \Delta G_{\text {hyd }}^{\text {exp }}(\mathrm{kcal} / \mathrm{mol})$ \\
\hline \hline $\mathrm{Li}^{+} \rightarrow \mathrm{Na}^{+}$ & 26.5 & {$[23.8 ; 26.2]$} \\
$\mathrm{Na}^{+} \rightarrow \mathrm{K}^{+}$ & 13.7 & {$[16.7 ; 17.7]$} \\
$\mathrm{K}^{+} \rightarrow \mathrm{Rb}^{+}$ & 3.2 & {$[4.9 ; 5.4]$} \\
$\mathrm{Rb}^{+} \rightarrow \mathrm{Cs}^{+}$ & 7.6 & {$[5.5 ; 7.7]$} \\
\hline $\mathrm{Li}^{+} \rightarrow \mathrm{Cs}^{+}$ & 51.4 & {$[50.9 ; 57.0]$} \\
\hline \hline $\mathrm{Mg}^{2+} \rightarrow \mathrm{Ca}^{2+}$ & 82.2 & {$[77.7 ; 80.3]$} \\
$\mathrm{Ca}^{2+} \rightarrow \mathrm{Sr}^{2+}$ & 25.3 & {$[29.8 ; 32.9]$} \\
\hline $\mathrm{Mg}^{2+} \rightarrow \mathrm{Sr}^{2+}$ & 107.8 & {$[107.5 ; 113.2]$} \\
\hline
\end{tabular}

TABLE X: Differences in Gibbs free energy of hydration: Simulated and experimental values. The experimental values for the monovalent ions are taken from references $\underline{79}-84$ and those for divalent ions from $81,83,84$.

The hydration free energy differences, for all the transmutations considered, are summarized in Tab. $\mathrm{X}$, together with the corresponding experimental values. The overall agreement with experiment is very good, with deviations never exceeding a few $\mathrm{kcal} / \mathrm{mol}$, and the large variations of $\Delta \Delta G_{\mathrm{hyd}}$ across the ion series being well reproduced. We note that some force fields are able to reproduce this quantity slightly more accurately, such as the non-polarizable

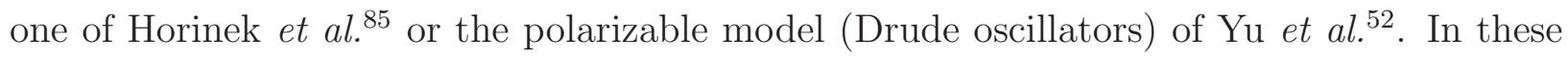
cases, however, experimental hydration free energies (or differences) were used as a target property to calibrate the force field, whereas we use it here as an independent validation of our ab-initio derived model.

\section{Diffusion coefficient}

The diffusion coefficient are computed using from the mean-squared displacement, as determined by the Einstein relation :

$$
D_{\mathrm{PBC}}=\lim _{t \rightarrow \infty} \frac{1}{6} \frac{\mathrm{d}\left\langle|\mathbf{r}(t)-\mathbf{r}(0)|^{2}\right\rangle}{\mathrm{d} t}
$$


The "PBC" subscript emphasizes the fact that the use of periodic boundary conditions induces a box length dependence on the measured diffusion coefficient, which takes the form 86 :

$$
D_{\mathrm{PBC}}=D_{0}-\frac{2.837 k_{B} T}{6 \pi \eta L}
$$

where $\eta$ is the shear viscosity of the solvent. For the box length of $L=18.65 \AA$ used in our simulations, the correction to the Dang-Chang water model is approximately $0.4310^{-9} \mathrm{~m}^{2} \mathrm{~s}^{-1}$ and must not be neglected $\left(D_{0}^{\mathrm{H}_{2} \mathrm{O}}=2.72 \pm 0.09 \mathrm{~m}^{2} \mathrm{~s}^{-1}\right)$. For a meaningful comparison with experiments, we thus extrapolate to the infinite box length limit, both for the ion and the water diffusion coefficients and compare the ratios $D_{0}^{\text {ion }} / D_{0}^{\mathrm{H}_{2} \mathrm{O}}$.

\begin{tabular}{|l|c|c|}
\hline Ion & $\left(D_{0}^{\text {ion }} / D_{0}^{\mathrm{H}_{2} \mathrm{O}}\right)^{\operatorname{sim}}$ & $\left(D^{\text {ion }} / D^{\mathrm{H}_{2} \mathrm{O}}\right)^{\exp }$ \\
\hline \hline $\mathrm{Li}^{+}$ & 0.49 & 0.44 \\
$\mathrm{Na}^{+}$ & 0.54 & 0.58 \\
$\mathrm{~K}^{+}$ & 0.78 & 0.88 \\
$\mathrm{Rb}^{+}$ & 0.88 & 0.90 \\
$\mathrm{Cs}^{+}$ & 0.82 & 0.89 \\
\hline \hline $\mathrm{Mg}^{2+}$ & 0.31 & 0.31 \\
$\mathrm{Ca}^{2+}$ & 0.35 & 0.34 \\
$\mathrm{Sr}^{2+}$ & 0.35 & 0.34 \\
\hline \hline $\mathrm{Cl}^{-}$ & 0.71 & 0.88 \\
\hline
\end{tabular}

TABLE XI: Ratio between the ion and water diffusion coefficients. The experimental values for the ions are taken from $\stackrel{88}{ }$, the one for the water from $\underline{89}$.

The simulation results are compared to the experimental ratios in Tab. XI. The relative error is of only 2 to $11 \%$ for the monovalent cations. The agreement is particularly good for the divalent cations, for which the relative error does not exceed 3\%. The largest relative error is for the $\mathrm{Cl}^{-}$ion (19\%). We performed similar simulations with the force field of Dang and co-workers $\underline{49}$, which uses the same water model. The errors in that case reach $31 \%$ for $\mathrm{Cl}^{-}$and $9 \%$ for $\mathrm{Ca}^{2+}$ and $\mathrm{Sr}^{2+}$. Moreover, while our results capture the equal diffusion coefficients of these two cations, the model of Dang and co-workers underestimates that of $\mathrm{Ca}^{2+}$ and overestimates that of $\mathrm{Sr}^{2+}$. 
Since force fields generally do not include experimental data on the dynamics for their calibration, their accuracy for dynamical properties is usually not as high as for structural and thermodynamic ones. The present strategy, which aims at reproducing the forces on the atoms and molecules, as best as possible, allows us to also predict the dynamic properties.

\section{E. Crystal density}

As a test of the interactions between ions, we now turn to the study of the ionic crystals. As explained above, out of the 241 parameters defining the force field for the entire family

of ions we have studied, only 3 (the dispersion damping parameters $b_{D}^{\mathrm{Cl}-\mathrm{Cl}}, b_{D}^{\mathrm{Cs}-\mathrm{Cl}}$ and $b_{D}^{\mathrm{Sr}-\mathrm{Cl}}$ ) were determined using the experimental densities of the 8 crystals as target properties. All the systems we studied preserved their correct crystal structure during the entire length of the simulations, even the more complex ones corresponding to the divalent cations. Fig. 6 illustrates the deformed rutile structure of $\mathrm{CaCl}_{2}$ and the lamellar one of $\mathrm{MgCl}_{2}$. While a complete study of the relative stability of the different possible phases exceeds the scope of the present work, this suggests that these phases are at least metastable. The simulated densities are compared to the experimental ones in Tab. XII, The overall agreement is once again good, with relative errors below $10 \%$ except for $\mathrm{NaCl}(16 \%)$.

\begin{tabular}{|c|c|c|}
\hline Crystal & $\rho_{\operatorname{sim}}\left(\mathrm{g} \cdot \mathrm{cm}^{-3}\right)$ & $\rho_{\exp }\left(\mathrm{g} \cdot \mathrm{cm}^{-3}\right)$ \\
\hline $\mathrm{LiCl}$ & 2.01 & 2.07 \\
\hline $\mathrm{NaCl}$ & 1.83 & 2.17 \\
\hline $\mathrm{KCl}$ & 1.93 & 1.99 \\
\hline $\mathrm{RbCl}$ & 2.98 & 2.76 \\
\hline $\mathrm{CsCl}$ & 4.42 & 3.99 \\
\hline $\mathrm{MgCl}_{2}$ & 2.21 & 2.33 \\
\hline $\mathrm{CaCl}_{2}$ & 2.04 & 2.15 \\
\hline $\mathrm{SrCl}_{2}$ & 3.25 & 3.05 \\
\hline
\end{tabular}

TABLE XII: Density of the crystals at 1 bar and $300 \mathrm{~K}$. The experimental values are taken from ${ }^{88}$. Note that the correct crystal structures (separated in the table) are preserved during the simulations. 


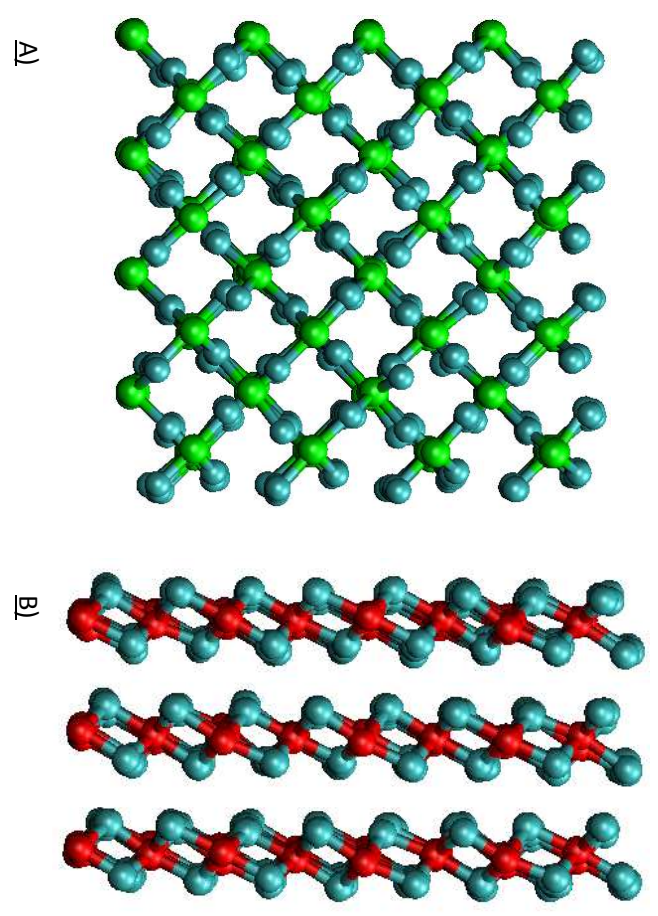

FIG. 6: A) Snapshot of $\mathrm{CaCl}_{2}$ cristal. B) Snapshot of $\mathrm{MgCl}_{2}$ cristal. Both structures are stable during the simulations. $\mathrm{Cl}^{-}$are in cyan, $\mathrm{Mg}^{2+}$ in red and $\mathrm{Ca}^{2+}$ in green.

Transferability to crystals is rarely tested, making comparisons with other potentials rather difficult. We have again used the force fields of Dang and coworkers $\underline{49}-51$ to assess the reliability of our potentials. Although their potentials give good results for $\mathrm{NaCl}$ $\left(\mathrm{d}=2.1\right.$ g.cm $\left.{ }^{-3}\right), \mathrm{KCl}\left(1.9\right.$ g.cm $\left.{ }^{-3}\right)$ and $\mathrm{CsCl}\left(3.8\right.$ g.cm $\left.{ }^{-3}\right)$ crystals, the structure proves to be unstable for $\mathrm{LiCl}, \mathrm{CaCl}_{2}$ and $\mathrm{SrCl}_{2}$. This example shows the need for more complicated force fields (with more parameters), as they can provide better transferability.

\section{F. Concentrated solutions}

The previous sections demonstrate the accuracy of the present force field for both infinitely dilute solutions and crystals. We now test its transferability to conditions which were not considered during the construction of the force field, by investigating concentrated 
ionic solutions. We compute the neutron diffraction spectra for concentrated $\mathrm{NaCl}$ and $\mathrm{KCl}$ solutions with one ion pair for 17 water molecules (1:17) from the site-site partial structure factor between site $\alpha$ and $\beta$ :

$$
S_{\alpha \beta}(Q)=4 \pi \rho \int r^{2}\left(g_{\alpha \beta}(r)-1\right) \frac{\sin (Q r)}{Q r} d r
$$

where $\rho$ is the atomic number density of the solution and $g_{\alpha \beta}(r)$ the corresponding site-site radial distribution function. Experimental neutron diffraction allows for the extraction of composite partial structure factors. We compare our simulations results to the traditionally used $F_{X X}$ function, defined as:

$$
F_{X X}(Q)=\frac{\sum_{\alpha, \beta}\left(2-\delta_{\alpha \beta}\right) c_{\alpha} c_{\beta} b_{\alpha} b_{\beta} S_{\alpha \beta}(Q)}{\left(\sum_{\alpha} c_{\alpha} b_{\alpha}\right)^{2}}
$$

where the sums over $\alpha$ and $\beta$ run over all atom types except hydrogen and $c_{\alpha}$ and $b_{\alpha}$ are the atomic fraction and neutron scattering length of atom $\alpha$, respectively. The comparison with the experimental results taken from Ref. $\underline{\underline{90}}$ in Figs. 7 and 8 indicates a very good agreement, which confirms the transferability to concentrated solutions.

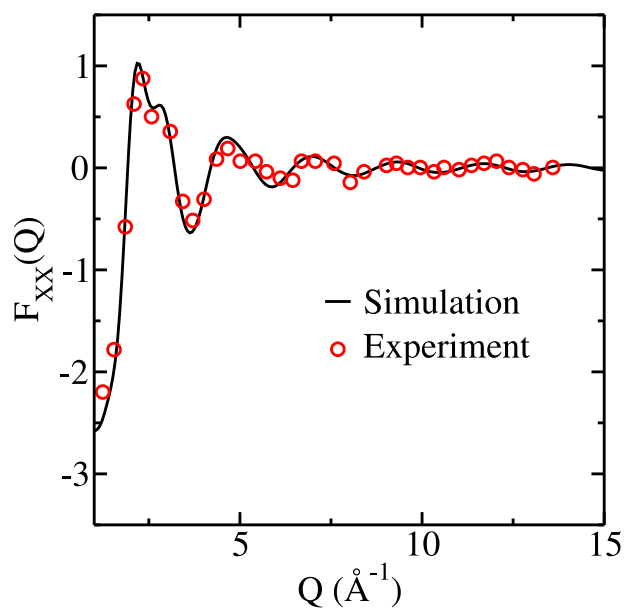

FIG. 7: Comparison between simulated and experimental $F_{X X}(Q)$ from Ref. $\underline{\underline{90}}$ for a concentrated $\mathrm{NaCl}$ solution (one $\mathrm{NaCl}$ pair for 17 water molecules).

\section{CONCLUSION}

We have shown a successful parametrization of a polarizable force field for aqueous solutions of $\mathrm{Li}^{+}, \mathrm{Na}^{+}, \mathrm{K}^{+}, \mathrm{Rb}^{+}, \mathrm{Cs}^{+}, \mathrm{Mg}^{2+}, \mathrm{Ca}^{2+}, \mathrm{Sr}^{2+}$ and $\mathrm{Cl}^{-}$ions. We used the polarizable 


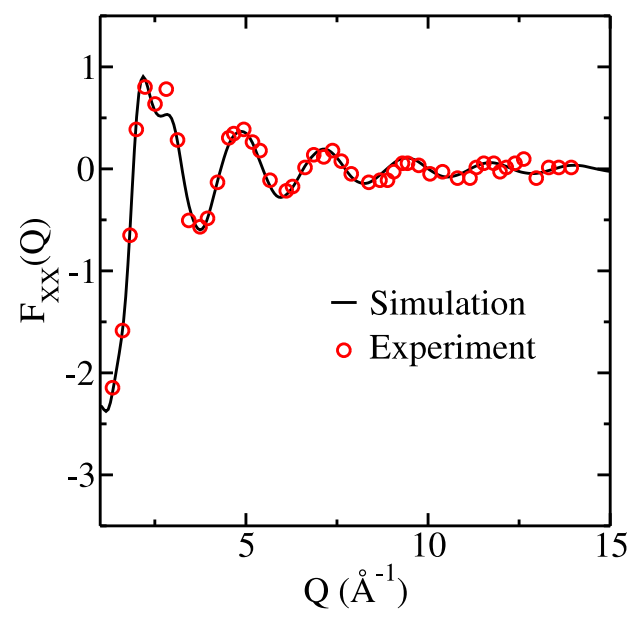

FIG. 8: Comparison between simulated and experimental $F_{X X}(Q)$ from Ref. $\underline{\underline{90}}$ for a concentrated $\mathrm{KCl}$ solution (one $\mathrm{NaCl}$ pair for 17 water molecules).

Dang-Chang model for water and derived all the parameters involving ions in the framework of the polarizable ion model of Madden and co-workers. The procedure relies only on ab-initio DFT calculations; part of the parameters (polarizabilities, dispersion coefficients) are directly calculated while the others are extracted from a generalized force- and dipole-matching procedure. Experimental information is used for validation purposes only: The structural (first-neighbour distances, coordination numbers), thermodynamic (hydration free energy differences) and dynamic (diffusion coefficients) are very well reproduced. The interactions between cations and the chloride anion are parametrized on calculations performed in the crystal phases, thus ensuring the accuracy of the force field across the whole concentration range.

The account of multi-body effects via the polarizability should ensure a good transferability to more complex conditions: mixtures of these salts, high temperature and pression $\underline{\underline{ }}$ and to liquid-vapor or liquid-solid interfaces. The next step will consist in extending the present approach to the interaction of water with the surface of oxide materials. It will then be possible to use this force field for the study of important problems of environmental science such as the retention of radionuclides onto clay minerals, or the water uptake by clays and zeolites. 


\section{Acknowledgments}

The authors acknowledge financial support from the Agence Nationale de la Recherche under grant ANR-09-SYSC-012 and from the Groupement National de Recherches PARIS. We also would like to thank Pr. Fabio Bruni and Pr. Alan K. Soper for providing us with the raw data of Ref. 90 .

1 J. Heyda, J. C. Vincent, D. J. Tobias, J. Dzubiella, and P. Jungwirth, J. Phys. Chem. B 114, $1213(2010)$.

2 H. I. Ingolfsson, Y. H. Li, V. V. Vostrikov, H. Gu, J. F. Hinton, R. E. Koeppe, and B. Roux, J. Phys. Chem. 115, 7417 (2011).

3 C. Domene, S. Vemparala, S. Furini, K. Sharp, and M. L. Klein, J. Am. Chem. Soc. 130, 3389 (2008).

4 P. Jungwirth and D. Tobias, Chem. Rev. 106, 1259 (2006).

5 B. Rotenberg, J.-P. Morel, V. Marry, P. Turq, and N. Morel-Desrosiers, Geochim. Cosmochim. Acta 73, 4034 (2009).

6 J.-P. Piquemal, L. Perera, G. A. Cisneros, P. Ren, L. G. Pedersen, and T. A. Darden, J. Chem. Phys. 125, 054511 (2006).

7 G. A. Tribello, F. Bruneval, C. Liew, and M. Parrinello, J. Phys. Chem. B 113, 11680 (2009).

8 T.-M. Chang and L. X. Dang, Chem. Rev. 106, 1305 (2006).

9 C. D. Wick, I.-F. W. Kuo, C. J. Mundy, and L. X. Dang, J. Chem. Theory Comput. 3, 2002 (2007).

10 A. Aguado, L. Bernasconi, S. Jahn, and P. A. Madden, Faraday Discuss. 124, 171 (2003).

11 D. Marrocchelli, M. Salanne, and P. Madden, J. Phys.: Condens. Matter 22, 152102 (2010).

12 M. Burbano, D. Marrocchelli, B. Yildiz, H. L. Tuller, S. T. Norberg, S. Hull, P. A. Madden, and G. W. Watson, J. Phys.: Condens. Matter 23 (2011).

13 S. Jahn and P. Madden, Phys. Earth Planet. Inter. 162, 129 (2007).

14 D. L. Newell, J. P. Kaszuba, H. S. Viswanathan, R. J. Pawar, and T. Carpenter, Geophys. Res. Lett. 35, L23403 (2008).

15 R. M. Espinosa-Marzal and G. W. Scherer, Acc. Chem. Res. 43, 897 (2010). 
16 M. A. Glaus, B. Baeyens, M. H. Bradbury, A. Jakob, L. R. Van Loon, and A. Yaroshchuk, Environ. Sci. Technol. 41, 478 (2007).

17 T. Gimmi and G. Kosakowski, Environ. Sci. Technol. 45, 1443 (2011).

18 W. Jorgensen, J. Chandrasekhar, J. Madura, R. Impey, and M. Klein, J. Chem. Phys. 79, 926 (1983).

19 H. Berendsen, J. Grigera, and T. Straatsma, J. Phys. Chem. 91, 6269 (1987).

20 J. L. F. Abascal and C. Vega, Phys. Rev. Lett. 98, 237801 (2007).

21 C. Vega and L. F. Abascal, Phys. Chem. Chem. Phys. 13, 19663 (2011).

22 P. J. van Maaren and D. van der Spoel, J. Phys. Chem. B 105, 2618 (2001).

23 T. Hansson, H. Yu, and W. F. van Gunsteren, J. Chem. Phys. 118, 221 (2003).

24 A. D. MacKerell, G. Lamoureux, and B. Roux, J. Chem. Phys. 119, 5185 (2003).

25 M. Sprik, J. Phys. Chem. 95, 2283 (1991).

26 S. J. Stuart, S. W. Rick, and B. J. Berne, J. Chem. Phys. 101, 6141 (1994).

27 J. Caldwell and P. Kollman, J. Phys. Chem. 99, 6208 (1995).

28 B. J. Berne and A. Wallqvist, J. Phys. Chem. A 97, 13941 (1993).

29 L. X. Dang and T.-M. Chang, J. Chem. Phys. 106, 8149 (1997).

30 A. A. Chialvo and P. T. Cumings, J. Chem. Phys. 105, 8274 (1996).

31 J. Sala, E. Guardia, and M. Masia, J. Chem. Phys. 133, 234101 (2010).

32 M. Masia, M. Probst, and R. Rey, J. Chem. Phys. 121, 7362 (2004).

33 M. Masia, M. Probst, and R. Rey, J. Chem. Phys. 123, 164505 (2005).

34 R. Heaton, R. Brookes, P. Madden, M. Salanne, C. Simon, and P. Turq, J. Phys. Chem. B 110, 11454 (2006).

35 N. Ohtori, M. Salanne, and P. Madden, J. Chem. Phys. 130, 104507 (2009).

36 N. Marzari and D. Vanderbilt, Phys. Rev. B 56, 12847 (1997).

37 B. Rotenberg, M. Salanne, C. Simon, and R. Vuilleumier, Phys. Rev. Lett. 104, 138301 (2010).

38 P. Madden, R. Heaton, A. Aguado, and S. Jahn, J. Mol. Struct.: THEOCHEM 771, 9 (2006).

39 M. Salanne, B. Rotenberg, S. Jahn, R. Vuilleumier, C. Simon, and P. A. Madden, Theor. Chem. Acc. 131, 1143 (2012).

40 K. Tang and J. Toennies, J. Chem. Phys. 80, 3726 (1984).

41 R. M. Martin, Electronic structure basic theory and practical methods (Cambridge University Press, 2004). 
J. J. Molina, S. Lectez, S. Tazi, M. Salanne, J. F. Dufreche, J. Roques, E. Simoni, P. A. Madden, and P. Turq, J. Chem. Phys. 134, 014511 (2011).

43 S. Grimme, J. Comput. Chem. 25, 1463 (2004).

44 P. L. Silvestrelli, Phys. Rev. Lett. 100, 053002 (2008).

45 Y. Andersson, D. C. Langreth, and B. I. Lundqvist, Phys. Rev. Lett. 76, 102 (1996).

46 R. O. Jones and O. Gunnarsson, Rev. Mod. Phys. 61, 689 (1989).

47 S. Kristyàn and P. Pulay, Chem. Phys. Lett. 229, 175 (1994).

48 A. J. Stone, Theory of intermolecular forces (Oxford University Press, Oxford, 1996).

49 L. X. Dang, J. Chem. Phys. 96, 6970 (1992).

50 T.-M. Chang and L. X. Dang, J. Phys. Chem. B 101, 10518 (1997).

51 L. X. Dang and T.-M. Chang, J. Phys. Chem. B 106, 235 (2002).

52 H. Yu, T. W. Whitfield, E. Harder, G. Lamoureux, I. Vorobyov, V. M. Anisimov, J. A. D. MacKerell, and B. Roux, J. Chem. Theory Comput. 6, 774 (2010).

53 A. Becke, Phys. Rev. A 38, 3098 (1988).

54 C. Lee, W. Yang, and R. Parr, Phys. Rev. B 37, 785 (1988).

55 J. Perdew, K. Burke, and M. Ernzerhof, Phys. Rev. Lett. 77, 3865 (1996).

56 N. Troullier and J. Martins, Phys. Rev. B 43, 001993 (1991).

57 S. Goedecker, M. Teter, and J. Hutter, Phys. Rev. B 54, 1703 (1996).

58 C. Hartwigsen, S. Goedecker, and J. Hutter, Phys. Rev. B 58, 1703 (1998).

59 M. Krack, Theor. Chem. Acc. 114, 145 (2005).

60 The CPMD consortium, CPMD version 3.13.2, URL http://www . cpmd.org.

61 CP2K developers group, URL http://cp2k.berlios.de.

62 F. James and M. Roos, Comp. Phys. Commun. 10, 343 (1975).

63 L. X. Dang, G. K. Schenter, V.-A. Glezakou, and J. L. Fulton, J. Chem. Phys. B 110, 23644 (2006).

64 L. X. Dang, G. K. Schenter, and J. L. Fulton, J. Phys. Chem. B 107, 14119 (2003).

65 A. Aguado and P. A. Madden, J. Chem. Phys. 119, 7471 (2003).

66 T. Laino and J. Hutter, J. Chem. Phys. 129, 074102 (2008).

67 G. Martyna, D. Tobias, and M. Klein, J. Chem. Phys. 101, 4177 (1994).

68 D. Jiao, C. King, A. Grossfield, T. A. Darden, and P. Ren, J. Phys. Chem. B 110, 18553 (2006).

69 S. Ramos, G. W. Neilson, A. C. Barnes, and M. J. Capitan, J. Chem. Phys. 118, 5542 (2003). 
70 P. R. Smirnov and V. N. Trostin, Rus. J. Phys. Chem 77, 2101 (2007).

71 H. Ohtaki and T. Radnal, Chem. Rev. 93, 1157 (1993).

72 D. Horinek, S. I. Mamatkulov, and R. R. Netz, J. Chem. Phys. 130, 124507 (2009).

73 Y. Marcus, Chem. Rev. 109, 1346 (2009).

74 J. J. Rehr, R. C. Albers, and S. I. Zabinsky, Phys. Rev. Lett. 69 (1992).

75 M. Kastenholz and P. Hunenberger, J. Chem. Phys. 124, 124106 (2006).

76 M. A. Kastenholz and P. Hunenberger, J. Chem. Phys. 124, 224501 (2006).

77 W. Press, B. Flannery, S. Teukolsky, and W. Vetterling, Numerical Recipes: the art of scientific computing (Cambridge University Press, 1992), 2nd ed.

78 F. B. Hildebrand, Introduction to numerical analysis (Dover publications, 1987).

79 M. D. Tissandier, K. A. Cowen, W. Y. Feng, E. Gundlach, M. H. Cohen, A. D. Earhart, and J. V. Coe, J. Phys. Chem. A 102, 7787 (1998).

80 J. E. B. Randles, Trans. Faraday Soc. 52, 1573 (1956).

81 R. Schmid, A. M. Miah, and V. N. Sapunov, Phys. Chem. Chem. Phys. 2, 97 (2000).

82 R. M. Noyes, J. Am. Chem. Soc. 84, 513 (1962).

83 R. Gomer and G. Tryson, J. Chem. Phys. 66, 4413 (1977).

84 Y. Marcus, J. Chem. Soc., Faraday Trans. 87, 2995 (1991).

85 D. Horinek, S. I. Mamatkulov, and R. R. Netz, J. Chem. Phys. 130 (2009).

86 I.-C. Yeh and G. Hummer, J. Phys. Chem. B 108, 15873 (2004).

87 S. Tazi, A. Botan, M. Salanne, V. Marry, P. Turq, and B. Rotenberg, J. Phys.: Condens. Matter in press.

88 D. R. Lide, ed., CRC Handbook of Chemistry and Physics (85th ed) (Taylor and Francis: Boca raton, FL, 2005).

89 K. Krynicki, C. D. Green, and D. W. Sawyer, Faraday Discuss. 66, 199 (1978).

90 R. Mancinelli, A. Botti, F. Bruni, M. A. Ricci, and A. K. Soper, Phys. Chem. Chem. Phys. 9, 2959 (2007).

91 S. Klotz, L. E. Bove, T. Strässle, T. C. Hansen, and A. M. Saitta, Nature Mater. 8, 405 (2009). 